\title{
Guest editorial to the theme issue on domain-specific modeling in theory and applications
}

\author{
Juha-Pekka Tolvanen · Matti Rossi · Jeff Gray
}

Published online: 17 February 2013

(C) Springer-Verlag Berlin Heidelberg 2013

\section{Introduction}

Interest in domain-specific modeling (DSM) comes from the aspiration to significantly improve the productivity and quality of software development by raising the level of abstraction beyond programming. This is done by specifying the solution directly using domain concepts, rather than lower level programming language and specific platform concepts that introduce layers of accidental complexity. In the past, productivity gains have been sought from new programming languages. Today, domain-specific modeling languages (DSMLs) provide a solution for continuing to raise the level of abstraction beyond coding, making development faster and easier.

In DSM, the models are constructed using concepts that represent things in the application domain, not concepts of a given programming language. The modeling language follows the domain abstractions and semantics, allowing developers to perceive themselves as working directly with domain concepts. The models represent simultaneously the design, implementation and documentation of the system (which can be generated directly from the models). In a number of cases, as some of the papers in this theme issue describe, the final products can be generated automatically from the high-level

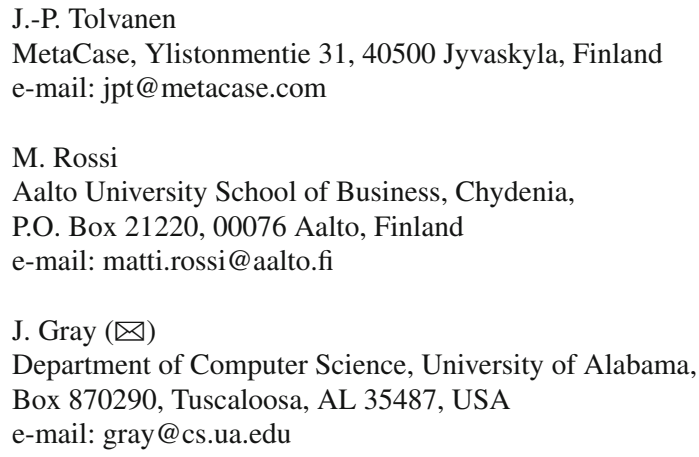

specifications with domain-specific code generators. This automation is possible because of domain-specificity: both the modeling language and code generators fit to the requirements of a narrowly defined domain.

This theme issue contains a collection of papers that represent advanced, state-of-the-art research in DSM, as well as new applications in practice. The selected papers also provide an overview of current open issues and identify potential lines for further research.

\section{Background context for this theme issue}

Much of the interest in DSM over the past decade has been captured in the archives of the annual DSM workshop at the OOPSLA/SPLASH conference series (http:// www.dsmforum.org/DSMworkshops.html), which averages between 35 and 50 attendees each year. Since 2001, authors have been publishing their papers in the DSM area at this workshop, which is now in its 12th year. Many of the submitting authors and reviewers have deep connections to this workshop and some of the ideas for papers in this theme issue had their genesis at this workshop. Prior to this theme issue in SoSyM, there were two other journal special issues related to the topics of DSM: a 2004 special issue in the Journal of Visual Languages and Computing, and a 2009 special issue of IEEE Software. This new SoSyM theme issue, as well as these other collections of DSM papers, represents much of the research contributions in DSM over the past decade.

\section{Selected papers for this theme issue}

We were overwhelmed by the response to the Call for Papers for this theme issue on "Domain-Specific Modeling 
in Theory and Applications." Overall, there were 59 full papers submitted, of which six were chosen for publication in this theme issue. There were two rounds of reviews and all papers were refereed by at least three well-known experts in the field. We are grateful for the large number of reviewers that did the hard work of evaluating the papers and suggesting improvements. The selection of the papers for this theme issue was assisted by the following reviewers: Pierre America, Robert Baillargeon, Krishnakumar Balasubramanian, Purushotham Bangalore, Peter Bell, Christoph Bockisch, Benjamin Braatz, Christoph Brandt, Jordi Cabot, Kai Chen, Tony Clark, Peter Clarke, Steve Cook, Diarmuid Corcoran, Dario Correal, Romain Delamare, Birgit Demuth, Dirk Derrider, Brandon Eames, Michael Golm, Øystein Haugen, Anne Haxthausen, Francisco Hernandez, Markus Herrmannsdörfer, James Hill, Pavel Hruby, Ethan Jackson, Jürgen Jung, Steven Kelly, Dimitris Kolovos, John Krogstie, Benoit Langlois, Florian Lautenbacher, Gunther Lenz, Mauri Leppänen, Tihamer Levendovszky, Johan Lilius, Juho Lindman, Shih-Hsi Liu, Henril Lönn, Kalle Lyytinen, Tomaz Lukman, Janne Merilinna, Marjan Mernik, Pedro J. Molina, Birger Moller-Pedersen, Andreas Opdahl, Juha Pärssinen, Kalle Piirainen, Pietu Pohjalainen, Ivan Porres, Mikko Riepula, Arturo Sanchez, David Servat, José Luis Sierra, Kari Smolander, Jonathan Sprinkle, Alin Stefanescu, Yu Sun, Tarja Systä, Dragos Truscan, Tuure Tuunanen, Andras Vadja, Hans Vangheluwe, Jules White, Christoph Wienands, Tau Yue, Jing Zhang and Steffen Zschaler.

The theme issue contains six articles dealing with DSM on both the theory and applications sides. The clear majority of the papers were dealing with different language implementations or requirements for DSM languages. Most of the papers also demonstrate the application of DSM or describe its use in practice. The papers included in this theme issue are as follows:

- The first paper, "Message Choreography Modeling" by Alin Stefanescu, Sebastian Wieczorek and Matthias Schur, presents a graphical DSML for Enterprise Service Integration. The work has close links to the needs of an industrial context, and the supporting tools have been applied in the context of SAP. The authors have applied the developed DSML in four pilots that all focus on real SOA-based products. The paper introduces different aspects of service choreographies, like message racing, global and local views, and message exchange viewpoints from an industrial perspective.

- The second paper, "Effective development of automation systems through domain-specific modeling in a small enterprise context" by Andrea Leitner, Christopher Preschern and Christian Kreiner, proposes an approach that allows small size companies to apply DSM. The viability of this incremental approach is demonstrated with two cases of automation system development. Because the authors have collected metrics on both cases, the benefits of DSM can be compared to other development approaches. The cases are good examples of generative development since in addition to code, also various other artifacts are generated, including hardware mapping, device configuration, web portal data, and various documentation for installation (e.g., wiring plans).

- The third paper, "Composing Domain-Specific Physical Models with General-Purpose Software Modules in Embedded Control Software" by Arjan de Roo, Hasan Sözer and Mehmet Aksit, discusses models for the embedded control domain. The paper combines a general-purpose language for implementing general computing tasks and a DSML for modeling the physical characteristics of the environment. The approach is demonstrated through industrial case studies.

- The fourth paper, "An Ontology-Based Framework for Domain-Specific Modeling" by Tobias Walter, Fernando Silva Parreiras and Steffen Staab, presents a framework for describing and reasoning about DSL's through ontology technologies. The framework allows debugging, commenting and progressive evaluation of metamodels under construction.

- In the fifth paper, "A Framework for Families of DomainSpecific Modelling Languages" by Benjamin Braatz and Christoph Brandt, describes how formal languages can be easier to accept and use by developers when there is a suitable DSM in place. This is demonstrated through an example of a resource description framework. The authors develop a small infrastructure language and demonstrate its use in an industrial development scenario.

- The concluding paper in the theme issue, entitled "DropsBox: The Dresden Open Software Toolbox", is written by Uwe Aßmann, Andreas Bartho, Christoff Bürger, Sebastian Cech, Birgit Demuth, Florian Heidenreich, Jendrik Johannes, Sven Karol, Jan Polowinski, Jan Reimann, Julia Schroeter, Mirko Seifert, Michael Thiele, Christian Wende and Claas Wilke. This paper introduces an open source toolbox for developing modeling tools within the Eclipse platform. The toolset can be customized to support different phases of the domain-specific language life cycle from language design to its use.

Acknowledgments We are thankful to all of those who submitted papers to this theme issue. We would also like to express our gratitude to the reviewers for the timely manner in which they assisted in choosing and making suggestions to improve the selected papers. We also would like to thank the SoSyM editorial office and, in particular, Martin Schindler, for the excellent support in preparing this theme issue. 


\section{Author Biographies}

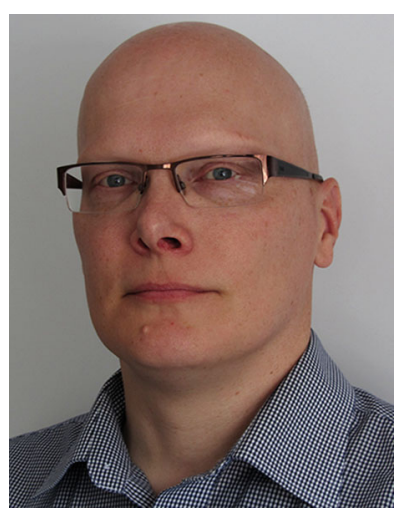

Juha-Pekka Tolvanen is the CEO of MetaCase and co-founder of the DSM Forum. He has been involved in model-driven development and tools, notably method engineering and metamodeling, since 1991. He has acted as a consultant worldwide for modeling language development, authored a book on Domain-Specific Modeling, and written over 70 articles for various software development magazines and conferences. Juha-Pekka co-started the OOPSLA workshops on DomainSpecific Modeling in 2001 and has been on the organizing committee since. He is also a member of IFIP WG8.1 Method Engineering task group and has recently co-edited a special Domain-Specific Modeling issue of IEEE Software. He is an adjunct professor at the University of Jyväskylä.

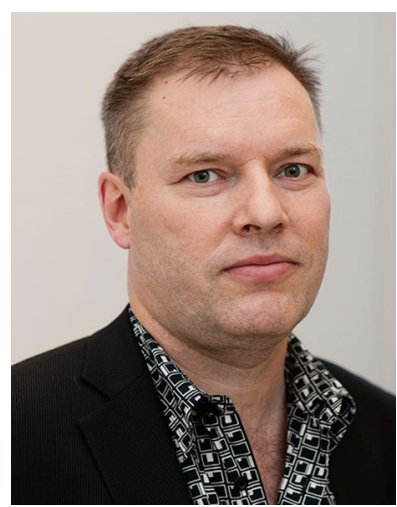

Matti Rossi is an associate professor in Information Systems Science in the Department of Information and Service Economy at the Aalto University School of Business. He has worked as research fellow at Erasmus University Rotterdam, visiting assistant professor at Georgia State University, Atlanta and visiting researcher at Claremont Graduate University. He has been the principal investigator in several major research projects funded by the technological development center of Finland and Academy of Finland. His research papers have appeared in journals such as MIS Quarterly, Journal of AIS, Information and Management andInformation Systems. He has been a senior editor of JAIS and Database, and he is the editor in chief of Communications of the Association for Information Systems. Matti Rossi is a member of IEEE, ACM and AIS.

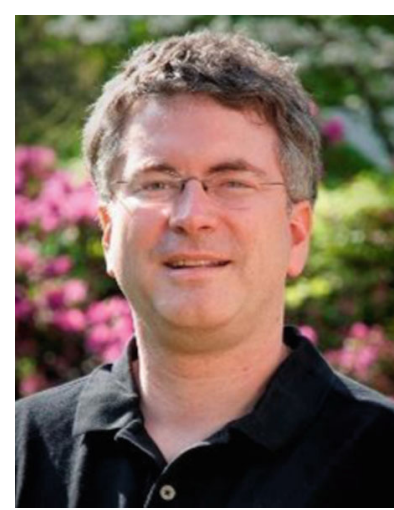

Jeff Gray is an associate professor in the Department of Computer Science at the University of Alabama where he co-directs the Software Engineering Group. His interests include model engineering, software maintenance, and computer science outreach to young children. Jeff is the recipient of an NSF CAREER Award and was named the Alabama Professor of the Year by the Carnegie Foundation (2008). He was Organizing Chair of AOSD 2009, Program co-Chair of SLE 2009, Program co-Chair of ICMT 2008, and Organizing co-Chair of MODELS 2013. Jeff is a Senior member of both the ACM and IEEE (serving as the Chair of the Alabama IEEE Computer Society). Additional information can be found at http://gray.cs.ua.edu. 